Rev. Adm. Saúde (On-line), São Paulo, v. 21, n. 82: e267, jan. - mar. 2021, Epub 31 mar. 2021 http://dx.doi.org/10.23973/ras.82.267

ARTIGO ORIGINAL

\title{
Instrumento norteador para auditoria clínica de protocolos
}

Guiding instrument for clinical audit of protocols

\section{Vitória Régia Dias dos Santos ${ }^{1}$, Tatiane Santos da Silva², Patrícia Gabriela Santana Alves ${ }^{2}$, Sara Diniz Rubinsztejn Azevedo ${ }^{3}$, Crystiane Aline Alves de Souza ${ }^{4}$, Eliana Cáceres dos Santos ${ }^{5}$, Rosemeire Keiko Hangai ${ }^{6}$}

1. Enfermeira. Aluna do Curso de Especialização em Administração Hospitalar do Programa de Estudos Avançados em Administração Hospitalar e de Sistemas de Saúde do Hospital das Clínicas da Faculdade de Medicina da Universidade de São Paulo (PROAHSA/HCFMUSP), São Paulo SP

2. Fisioterapeuta. Aluna do Programa de Residência Multiprofissional em Gestão Integrada de Sistemas de Saúde (COREMU/HCFMUSP), São Paulo SP

3. Coordenadora da Assessoria de Qualidade e Gerência de Risco do Instituto da Criança (ICr) do HCFMUSP, São Paulo SP

4. Gerente de Risco ICr/HCFMUSP, São Paulo SP

5. Analista de Qualidade ICr/HCFMUSP, São Paulo SP

6. Enfermeira. Coordenadora de ensino do PROAHSA/HCFMUSP, São Paulo SP

\section{RESUMO}

A auditoria clínica envolve um processo de verificação das práticas preconizadas e o cuidado prestado nas instituições de saúde implicando na qualidade do atendimento, neste contexto o trabalho tem como objetivo elaborar um protótipo, avaliar aplicabilidade e oportunidades de melhoria de um instrumento norteador para análise da eficácia da implantação de protocolos. Realizado em hospital pediátrico de ensino de grande porte entre abril e setembro de 2020, no município de São Paulo, através de levantamento 
bibliográfico e de protocolos institucionais, interações com profissionais da Instituição e um teste-piloto. O instrumento em sua versão validada foi baseado na estrutura comum de seis protocolos, constando de seis critérios correspondentes ao objetivo, abrangência, fluxo de atendimento, condutas adotadas, registros em prontuário e indicadores sendo as informações classificadas em conforme, parcialmente e não conforme. No processo de validação verificou a necessidade de instrumento específico para cada protocolo auditado. O instrumento elaborado atendeu ao objetivo proposto de nortear a prática de auditoria dentro do hospital e demonstrou conseguir em conjunto com específico agregar valor ao atendimento apontando possíveis melhorias.

Palavras-chave: Auditoria Clínica; Qualidade da Assistência à Saúde; Indicadores de Qualidade em Assistência à Saúde; Protocolos Clínicos

\begin{abstract}
The clinical audit involves a process of verifying the recommended practices and the are provided in health institutions, implying in the quality of care, in this context the work aims to develop a prototype, evaluate applicability and opportunities for improvement of a guiding instrument for analyzing the effectiveness the implementation of protocols. Held in a large teaching pediatric hospital between April and September 2020, in the city of São Paulo, through a bibliographic survey and institutional protocols, interactions with professionals of the Institution and a pilot test. The instrument in its validated version was based on the common structure of six protocols, consisting of six criteria corresponding to the objective, scope, service flow, conduct adopted, medical records and indicators being classified as conforming, partially and nonconforming. In the validation process, he verified the need for a specific instrument for each audited protocol. The instrument developed met the proposed objective to guide the audit practice within the hospital and obtain the set with the specific value of adding possible improvements to care.
\end{abstract}

Keywords: Clinical Audit; Quality of Health Care; Quality Indicators, Health Care; Clinical Protocols

\title{
INTRODUÇÃO
}

Em 2002, a Organização Mundial de Saúde (OMS) publicou a resolução WHA 55.18 alertando para necessidade de colocar em foco a segurança do paciente nos serviços de saúde ${ }^{1}$. 
Nesse contexto a auditoria clínica surge como forma de garantir a qualidade do cuidado prestado através de processo sistemático para avaliar o que está sendo realizado com o que foi estabelecido em termos de melhores práticas institucionais e levantar oportunidades de melhoria para atendimento aos pacientes ${ }^{2,3}$.

A ideia acima é referenciada no documento Principles for Best Practice in Clinical Audit (2002) traz a definição de auditoria clínica do National Health Service (NHS) apoiado pelo National Institute for Clinical Excellence (NICE) como processo de melhoria da qualidade com foco no atendimento e resultados aos pacientes através de revisão sistemática do realizado na prática assistencial com estabelecido em critérios explícitos ${ }^{4}$.

Como benefícios a auditoria clínica possibilita comparação com outros serviços, melhoria da qualidade dos serviços e criação de conjunto de dados para permitir o processo de comparação, a promoção da prática esperada, provê oportunidades para desenvolvimento da equipe assistencial e melhorias na prestação da assistência e desfechos para o paciente ${ }^{2,3}$.

Considerando o contexto de verificação da adoção de padrões de cuidado adotados pelos serviços em saúde faz parte do objeto de trabalho da auditoria clínica a verificação da conformidade dos protocolos.

Os protocolos clínicos tendem a aprimorar a assistência, pois são baseados em dados científicos e buscam padronizar os tipos de tratamento e condutas para determinadas situações com bases nas melhores evidências científicas, seu processo de auditoria releva oportunidades para mudanças ou manutenção e necessidade de treinamentos dos profissionais envolvidos na assistência direta 5 .

No processo de aprimoramento dos cuidados prestados, a instituição objeto deste trabalho viu a oportunidade de aperfeiçoar o acompanhamento da eficácia de uso dos protocolos para garantir prestação de atendimento de excelência a seus usuários através de ferramenta de apoio ao processo de auditoria clínica.

Diante do citado, o objetivo deste trabalho foi elaborar um protótipo, avaliar aplicabilidade e oportunidades de melhoria de um instrumento norteador para análise da eficácia da implantação de protocolos.

\section{METODOLOGIA}

Trata-se de um trabalho de desenho exploratório de caráter qualitativo através de procedimentos técnicos bibliográficos e levantamento de informações ${ }^{6}$.

Teve como cenário um hospital pediátrico de ensino de grande porte, localizado no município de São Paulo. Instituição inaugurada em 1976 que atende público recém-nascido, criança e adolescente em diversas especialidades com foco na alta complexidade, com usuários do sistema de saúde público e suplementar sendo referência nacional em saúde infantil. 
O processo de desenvolvimento e validação ocorreu no período de 27 de abril a 17 de setembro de 2020 e incluíram reuniões com responsáveis da área de qualidade da Instituição, profissionais da assistência, levantamento de seis protocolos institucionais e desenvolvimento de instrumentais com software Microsoft Excel.

Além disso, foi levantado material bibliográfico através das plataformas CAPES, Cochrane, PubMed, SciELO e Google Acadêmico usando os descritores auditoria, auditoria clínica, protocolo e jejum utilizando as técnicas booleanas and e or com período de busca entre 2008 a 2020 abrangendo os idiomas português e inglês.

Para piloto foi selecionado o protocolo de abreviação de jejum da Instituição, com auditoria de 34 prontuários entre agosto de 2018 a setembro de 2020 contemplando unidade de internação e de cirurgia.

Contudo por questões éticas e de respeito aos pacientes este trabalho restringe-se a relatar o desenvolvimento da ferramenta, suas necessidades de modificação, criação de instrumentos acessórios e resultado da auditoria, sem permear aos detalhes dos resultados obtidos com auditoria dos prontuários.

\section{RESULTADOS}

Para elaboração do protótipo do instrumento norteador foram levantados seis protocolos institucionais (Tabela 1). A partir da análise da estrutura de cada um foi elaborado um padrão dos itens comuns, sendo elencados os itens: definição, objetivos, abrangência, critérios, condutas, indicadores e documentos.

Tabela 1. Protocolos analisados e estrutura comum.

\begin{tabular}{|l|}
\hline Temática dos protocolos estudados \\
\hline Algoritmo para a Avaliação e Tratamento da Dor \\
\hline $\begin{array}{l}\text { Inserção, Manipulação e Manutenção de Cateter Central de Inserção } \\
\text { Periférica }\end{array}$ \\
\hline $\begin{array}{l}\text { Prevenção e Manejo das Infecções de Corrente Sanguínea Associadas ao } \\
\text { Cateter Venoso Central }\end{array}$ \\
\hline Antibioticoprofilaxia em Cirurgia \\
\hline $\begin{array}{l}\text { Prevenção e Manejo de Pneumonia Associada à Ventilação Mecânica do } \\
\text { Paciente Pediátrico }\end{array}$ \\
\hline Transplante Hepático Pediátrico \\
\hline
\end{tabular}

Após levantamento dos sete itens comuns para os protocolos foram elaborados os critérios norteadores, em um formulário no Excel, apresentados na Tabela 2. 
Para indicação de conformidade foi adotando um sistema binário para classificação onde 0 significava não conforme e 1 conforme.

Tabela 2. Critérios elaborados para avaliação de aplicação de protocolos

\begin{tabular}{|l|}
\hline Critério \\
\hline $\begin{array}{l}\text { A aplicação do protocolo atingiu os objetivos descritos em documento } \\
\text { institucional }\end{array}$ \\
\hline O paciente estava dentro da abrangência do protocolo \\
\hline Existe justificativa clínica para inserção no protocolo \\
\hline $\begin{array}{l}\text { O paciente foi adequadamente inserido no protocolo, conforme critérios de } \\
\text { inclusão }\end{array}$ \\
\hline As condutas adotadas no atendimento respeitaram os padrões do protocolo \\
\hline É possível verificar em prontuário os fluxos de atendimento \\
\hline $\begin{array}{l}\text { As adoções de condutas indicadas no protocolo estão registradas em } \\
\text { prontuário }\end{array}$ \\
\hline $\begin{array}{l}\text { Os resultados clínicos são coerentes com os padrões de cuidado do } \\
\text { protocolo }\end{array}$ \\
\hline Dados em prontuário permitem calcular os indicadores \\
\hline
\end{tabular}

Ao final desta etapa de prototipação foi elaborada planilha usando recurso de lista suspensa para seleção dos protocolos e dos valores binários e préformatada para realizar a soma das pontuações e destaque em vermelho para valores iguais ou abaixo de 4, indicando número alto de itens não conformes. Essa identificação trata de um alerta para o avaliador verificar gaps de aplicação do protocolo quanto aos critérios elaborados.

Para acompanhar o resultado geral da avaliação foi elaborado o índice de conformidade com protocolo que refere ao percentual de atendido pelo número de critérios levantados.

$\mathrm{Na}$ etapa de validação foi selecionado o protocolo de abreviação de jejum para piloto da ferramenta que antes passou por revisão pelos profissionais responsáveis da área de qualidade do hospital.

A revisão resultou em alterações referentes aos critérios elaborados com redução no número de critérios de 9 para 6 , as alterações e argumentações estão presentes nas Tabelas 3 e 4.

Tabela 3. Alterações de critérios pós-validação

\begin{tabular}{|l|l|l|}
\hline Alteração & Motivo & $\begin{array}{l}\text { Critério pós- } \\
\text { validação }\end{array}$ \\
\hline
\end{tabular}




\begin{tabular}{|l|l|l|}
\hline $\begin{array}{l}\text { Unificação dos } \\
\text { critérios 2, 3 e } 4\end{array}$ & $\begin{array}{l}\text { Similares no item avaliado, pois } \\
\text { todos os critérios referiam à } \\
\text { abrangência do protocolo }\end{array}$ & $\begin{array}{l}\text { O paciente estava } \\
\text { dentro da abrangência } \\
\text { do protocolo }\end{array}$ \\
\hline $\begin{array}{l}\text { Unificação dos } \\
\text { critérios } 5 \text { e } 7\end{array}$ & $\begin{array}{l}\text { Itens referem à avaliação da } \\
\text { mesma estrutura comum, } \\
\text { aplicação da conduta do } \\
\text { protocolo e registro em prontuário }\end{array}$ & $\begin{array}{l}\text { As adoções de } \\
\text { condutas indicadas no } \\
\text { protocolo estão } \\
\text { registradas em } \\
\text { prontuário }\end{array}$ \\
\hline
\end{tabular}

Tabela 4. Critérios norteadores pós-validação

1. A aplicação do protocolo atingiu os objetivos descritos em documento institucional

2. O paciente estava dentro da abrangência do protocolo

3. É possível verificar em prontuário os fluxos de atendimento

4. As condutas adotadas no atendimento respeitaram os padrões do protocolo

5. As adoções de condutas indicadas no protocolo estão registradas em prontuário

6. Dados em prontuário permitem calcular os indicadores

Além da alteração supracitada, foi realizada a inclusão da pontuação parcialmente conforme, visto que para a avaliação de alguns itens, muitos pontos devem ser avaliados, sendo alguns destes parcialmente conforme.

A pontuação parcialmente conforme foi considerada quando 2 ou mais pontos conformes na avaliação de um item, pôr fim a distribuição das pontuações seguiu o conforme 2 pontos, parcialmente conforme 1 e não conforme 0.

A unificação dos critérios demonstrou necessidade de uma reestruturação da pontuação e do índice de conformidade do instrumento passando com pontuação máxima de 12 pontos e pontuação mínima de 6 pontos para a interpretação positiva da eficácia da implantação dos protocolos.

Durante processo de validação tendo como base o protocolo de abreviação de jejum foi verificado a necessidade de criação de checklist específico com objetivo de guiar o auditor nos critérios preconizados no instrumento norteador. Dessa forma, otimizando o processo de auditoria do protocolo pela disposição dos itens que devem ser avaliados, sendo estruturado e organizado conforme os itens do instrumento norteador a fim de facilitar a transposição dos dados para análise do índice de conformidade, tal necessidade já era prevista considerando a configuração genérica do instrumento. 
Além da elaboração de checklist específico, foi realizado o mapeamento do local no prontuário eletrônico do paciente (PEP) que apresentava as informações necessárias para atender a ferramenta, conforme a Tabela 5.

Tabela 5. Descrição dos locais a serem consultados no PEP para informações de auditoria clínica

\begin{tabular}{|c|c|c|}
\hline $\begin{array}{l}\text { Critérios } \\
\text { norteadores }\end{array}$ & $\begin{array}{l}\text { Itens do checklist } \\
\text { específico }\end{array}$ & Local de busca no PEP \\
\hline Objetivo & $\begin{array}{l}\text { Descrição de fome, sede } \\
\text { e/ou ansiedade }\end{array}$ & Anotação de enfermagem \\
\hline \multirow[t]{2}{*}{ Abrangência } & $\begin{array}{l}\text { Informações do } \\
\text { procedimento cirúrgico } \\
\text { (tipo, data e horário) }\end{array}$ & $\begin{array}{l}\text { Histórico do Adendo de } \\
\text { internação; } \\
\text { Documentos de enfermagem } \\
\text { pré-operatório: SAEP- registro } \\
\text { transoperatório e checklist de } \\
\text { segurança para o paciente } \\
\text { cirúrgico - recepção do } \\
\text { paciente }\end{array}$ \\
\hline & Critérios de exclusão & $\begin{array}{l}\text { Adendo de internação; } \\
\text { Prescrição médica; } \\
\text { Prescrição de enfermagem; } \\
\text { Anotação de enfermagem; } \\
\text { Balanço hídrico }\end{array}$ \\
\hline $\begin{array}{l}\text { Fluxo de } \\
\text { atendimento }\end{array}$ & $\begin{array}{l}\text { Fluxo das unidades de } \\
\text { atendimento do paciente }\end{array}$ & $\begin{array}{l}\text { Anotação de enfermagem; } \\
\text { Adendo de internação }\end{array}$ \\
\hline \multirow[t]{5}{*}{$\begin{array}{l}\text { Conduta, registro } \\
\text { e Indicadores }\end{array}$} & Via de alimentação & $\begin{array}{l}\text { Balanço hídrico; } \\
\text { Nutrição: orientação nutricional } \\
\text { e evolução dietoterápica } \\
\text { diária; } \\
\text { Anotação de enfermagem }\end{array}$ \\
\hline & Peso & $\begin{array}{l}\text { Avaliação antropométrica de } \\
\text { nutrição } \\
\text { Balanço hídrico }\end{array}$ \\
\hline & $\begin{array}{l}\text { Quantidade de líquido } \\
\text { ofertado }\end{array}$ & $\begin{array}{l}\text { Anotação de enfermagem; } \\
\text { Balanço hídrico agrupado }\end{array}$ \\
\hline & Tempo total de jejum & $\begin{array}{l}\text { Checklist de segurança para o } \\
\text { paciente cirúrgico; } \\
\text { Evolução Dietoterápica Diária; } \\
\text { Anotação de enfermagem }\end{array}$ \\
\hline & $\begin{array}{l}\text { Oferta de líquidos claros } \\
\text { (tipo, quantidade ofertada e }\end{array}$ & Anotação de enfermagem; \\
\hline
\end{tabular}




\begin{tabular}{|l|l|l|}
\hline $\begin{array}{l}\text { quantidade aceita pelo } \\
\text { paciente) }\end{array}$ & $\begin{array}{l}\text { Prescrição de enfermagem; } \\
\text { Balanço hídrico; } \\
\text { Prescrição médica; } \\
\end{array}$ \\
& $\begin{array}{l}\text { Doc. de nutrição; } \\
\text { Sistematização de Assistência } \\
\text { de Enfermagem }\end{array}$ \\
\hline
\end{tabular}

O instrumento geral e específico para protocolo de jejum foi aplicado em 34 prontuários utilizando as versões primária e validada pela equipe do serviço do instrumento criado. Para versão primária foi alcançado um grau de conformidade foi de $42 \%$ enquanto para versão validada este foi de $48 \%$, esta diferença se deve ao acréscimo da categoria parcialmente conforme e consequente alteração na pontuação.

Foi adotado a versão validada pelo seu maior nível de detalhes na pontuação das informações levantadas em prontuários.

\section{DISCUSSÃO}

Para elaboração dos critérios norteadores com base nos protocolos foram adotadas as etapas para planejamento de auditoria ${ }^{7}$ do Quadro 1, características de um processo de trabalho de auditoria ${ }^{2}$ e orientações para perguntas de auditoria ${ }^{8}$.

Quadro 1. Etapas para planejamento de auditoria.

\begin{tabular}{|l|l|}
\hline Etapas & Respostas do estudo \\
\hline O que? & Protocolos institucionais \\
\hline Por quê? & Avaliar eficácia dos protocolos institucionais \\
\hline Quem? & Pacientes \\
\hline Onde? & Nas unidades de atendimento \\
\hline Quando? & Periodicamente \\
\hline Como? & Formulário e leitura de prontuário \\
\hline
\end{tabular}

O reconhecimento da qualidade da assistência passa por três dimensões: segurança do paciente, experiência do paciente e eficácia do atendimento. A segurança do paciente está relacionada ao gerenciamento de riscos com mitigação das ameaças associadas ao cuidado. A experiência trata da expressão do paciente quanto ao atendimento recebido e, por fim, a eficácia do atendimento possibilita julgar se os profissionais estão realizando as coisas da maneira certa para alcançar os objetivos esperados ${ }^{8}$. 
De acordo com a literatura as orientações para perguntas a serem realizadas em uma auditoria devem envolver então a segurança, experiência do paciente e efeito terapêutico do cuidado prestado. É importante que as informações sejam coletadas de todos os interessados, e os questionamentos permitam a descrição do serviço, a quem se destina como é experimentado e como os profissionais o entregam ${ }^{8}$.

Foram adotadas as características de um processo de trabalho para ser considerado auditoria preconizadas na literatura ${ }^{2}$ e apresentadas no Quadro 2.

Quadro 2. Características de um processo de trabalho de auditoria.

Condução para produzir informações que visem melhorar a assistência fornecida

Ter como foco a resposta do serviço à meta de um padrão pré-determinado

Não requerer comitê de ética em pesquisa

Conforme a literatura a realização de uma auditoria trata de um processo cíclico denominado de ciclo de auditoria clínica ou espiral, iniciando pelo levantamento dos tópicos que serão auditados e a viabilidade da auditoria. $O$ foco do processo é sempre repercutir para melhoria da qualidade do serviço visando práticas a serem aprimoradas ou verificação de padrões de desempenho. Em seguida são levantados os aspectos a serem incluídos na auditoria através de documentos ou parâmetros existentes 7,9 .

Em uma terceira etapa é recomendado elaboração de critérios e padrões para auditoria a ser realizada. Critérios representando as afirmações que definem as boas práticas e os padrões o grau percentual de atendimento a esses critérios pela instituição auditada ${ }^{7,9}$.

O trabalho alcançou a delimitação do objeto de auditoria como sendo os protocolos da Instituição e os aspectos a serem considerados de sua estrutura comum, com viabilidade de realização através de uma ferramenta elaborada em software amplamente utilizado e estabelecimento de critérios a serem atingidos.

Ainda de acordo com a literatura, o ciclo passa a uma quarta etapa com a realização da coleta de dados. Nesse momento deve ser proposta a ferramenta para coleta dos dados. Em geral, essa coleta ocorre de forma manual com formulários ou utilizando softwares para seu registro. Na etapa seguinte é realizada a análise dos dados tendo por objetivo a comparação entre o apresentado na realidade com os critérios e padrões definidos 7,9 .

No caso em questão foram elaboradas ferramentas no software Excel ${ }^{\circledR}$ para coleta dos dados com posterior análise para comparação entre realidade e $o$ preconizado, assim o trabalho alcançou as cinco etapas do ciclo de auditoria. 
As etapas subsequentes para completar o ciclo de oito são referentes à implantação das mudanças encontradas com realização de uma nova auditoria para comparação dos achados antes e pós-implantação das mudanças e elaboração do relatório de auditoria para registro e compartilhamento de informações ${ }^{7,9}$.

O estudo atendeu o ciclo até etapa cinco, sendo as demais representadas na entrega de relatório ao hospital objeto do estudo apresentando as oportunidades encontradas.

\section{CONCLUSÃO}

O instrumento mostrou atender a uma avaliação genérica passível de ser aplicada para qualquer protocolo da Instituição, contudo sendo necessária a criação de um bloco específico para cada protocolo analisado. A aplicação antes e após alterações demonstrou redução dos itens a serem analisados atendeu a estrutura dos protocolos vigentes e alcançou os objetivos.

Demonstrou responder a necessidade de acompanhamento da qualidade do serviço e proporcionou conhecer oportunidades de melhoria na aplicação do protocolo de abreviação de jejum do hospital quanto a treinamento, sensibilização e mudanças de fluxos.

Recomenda-se sua aplicação aos demais protocolos institucionais englobando protocolos clínicos, preventivos e de segurança dos pacientes e criação de equipe multiprofissional para auditoria.

\section{REFERÊNCIAS}

1. Brasil. Agência Nacional de Vigilância Sanitária. Gestão de Riscos e Investigação de Eventos Adversos Relacionados à Assistência à Saúde. Brasília; 2017. Disponível em: https://www20.anvisa.gov.br/segurancadopaciente/index.php/publicacoes/ite $\mathrm{m} /$ caderno-7-gestao-de-riscos-e-investigacao-de-eventos-adversosrelacionados-a-assistencia-a-saude.

2. Clinical Audit Support Centre [internet]. What is Clinical Audit.Leicester, 2018. Disponível em: http://www.clinicalauditsupport.com/what-is-clinicalaudit.html

3. Viana CS. O papel da auditoria nas instituições hospitalares. Revista Científica Multidisciplinar Núcleo do Conhecimento [internet]. 2019;04(11):05-20. Disponível em: https://www.nucleodoconhecimento.com.br/administracao/papel-daauditoria. 
4. National Institute for Clinical Excellence [internet]. Principles for Best Practice in Clinical Audit. Abingdon: Radcliffe Medical Press; 2002.

Disponível em: https://www.nice.org.uk/media/default/About/what-wedo/Into-practice/principles-for-best-practice-in-clinical-audit.pdf

5. Pimenta CAMP et al. Guia para a Implementação de Protocolos Assistenciais de Enfermagem: integrando protocolos, prática baseada em evidência e classificações de enfermagem. São Paulo: Coren-SP; 2017. Disponível em: https://portal.coren-sp.gov.br/wpcontent/uploads/2010/01/guia implementacao protocolos assistenciais enf ermagemintegrando protocolos pratica baseada em evidencia classificacao enfer $\underline{\text { magem.pdf }}$

6. Creswell JW. Procedimentos qualitativos. In.: Projeto de pesquisa: Método quantitativo, qualitativo e misto. $2^{\underline{a}}$ ed. Porto Alegre: Ed. Artmed; 2007.

7. Grainger A. Clinical audit: shining a light on good practice. Nurs. Manage [internet]. 2010; 17(4):30-33. Disponível em: https://rcnpublishing.com/doi/abs/10.7748/nm2010.07.17.4.30.c7847

8. Patel S. Identifying best practice principles of audit in health care. Nurs. Stand [internet]. 2010; 24(32):40-48. Disponível em: https://rcnpublishing.com/doi/abs/10.7748/ns2010.04.24.32.40.c7713

9. Ashmore S, Ruthven T. Clinical audit: a guide. Nurs. Manage [internet]. 2008; 15(1):18-22. Disponível em: https://journals.rcni.com/nursingmanagement/clinical-audit-a-guide-nm2008.04.15.1.18.c6489

Recebido: 10 de fevereiro de 2021. Aceito: 31 de março de 2021

Correspondência: Vitória Régia Dias dos Santos Cavalcante. E-mail: vitoriaregiasantos@hotmail.com

Conflito de Interesses: os autores declararam não haver conflito de interesses. 